\title{
Visual Working Memory Capacity and the Medial Temporal Lobe
}

\author{
Annette Jeneson, ${ }^{1}$ John T. Wixted, ${ }^{1}$ Ramona 0 . Hopkins, ${ }^{4,5}$ and Larry R. Squire ${ }^{1,2,3,6}$ \\ Departments of ${ }^{1}$ Psychology, ${ }^{2}$ Psychiatry, and ${ }^{3}$ Neurosciences, University of California, San Diego, California 92093, ${ }^{4}$ Department of Psychology and \\ Neuroscience Center, Brigham Young University, Provo, Utah 84143, ${ }^{5}$ Department of Medicine, Pulmonary and Critical Care Division, Intermountain \\ Medical Center, Murray, Utah 84143, and ${ }^{6}$ Veterans Affairs Medical Center, San Diego, California 92161
}

Patients with medial temporal lobe (MTL) damage are sometimes impaired at remembering visual information across delays as short as a few seconds. Such impairments could reflect either impaired visual working memory capacity or impaired long-term memory (because attention has been diverted or because working memory capacity has been exceeded). Using a standard change-detection task, we asked whether visual working memory capacity is intact or impaired after MTL damage. Five patients with hippocampal lesions and one patient with large MTL lesions saw an array of 1,2,3, 4, or 6 colored squares, followed after 3, 4, or 8 s by a second array where one of the colored squares was cued. The task was to decide whether the cued square had the same color as the corresponding square in the first array or a different color. At the $1 \mathrm{~s}$ delay typically used to assess working memory capacity, patients performed as well as controls at all array sizes. At the longer delays, patients performed as well as controls at small array sizes, thought to be within the capacity limit, and worse than controls at large array sizes, thought to exceed the capacity limit. The findings suggest that visual working memory capacity in humans is intact after damage to the MTL structures and that damage to these structures impairs performance only when visual working memory is insufficient to support performance.

\section{Introduction}

A fundamental distinction can be drawn between immediate memory or working memory on the one hand, and long-term memory on the other. When material is presented for learning, a limited amount of information can be held in immediate memory and actively maintained in working memory (Baddeley and Hitch, 1974). Long-term memory refers to what can be recalled when the information is no longer actively maintained, either because immediate memory capacity was exceeded or because attention was diverted from the memoranda (Drachman and Arbit, 1966).

Early studies of memory-impaired patients with medial temporal lobe (MTL) damage suggested that MTL structures are involved in the formation of long-term memory and that immediate memory and working memory are independent of these structures (Drachman and Arbit, 1966; Baddeley and Warrington, 1970; Milner, 1972). Yet, recent observations of impaired performance in MTL patients on some short-delay tasks involving visual or relational information, and fMRI studies of MTL activity in healthy individuals performing sim-

\footnotetext{
Received Dec. 23, 2011; revised Jan. 20, 2012; accepted Jan. 26, 2012.

Author contributions: A.J. and L.R.S. designed research; A.J. performed research; R.O.H. contributed unpublished reagents/analytic tools; A.J. analyzed data; A.J., J.W., and L.R.S. wrote the paper.

This work was supported by the Medical Research Service of the Department of Veteran Affairs and National Institute of Mental Health Grant MH24600. We thank Jennifer Frascino, Anna van der Horst, Ashley Knutson, Kristin Mauldin, and Zhuang Song for assistance. We also thank Steven Hillyard and John Serences for helpful discussion.

Correspondence should be addressed to Dr. Larry R. Squire, Veterans Affairs Medical Center 116A, 3550 La Jolla Village Drive, San Diego, CA 92161. E-mail: Isquire@ucsd.edu.

DOI:10.1523/JNEUROSCI.6444-11.2012

Copyright $\odot 2012$ the authors $\quad 0270-6474 / 12 / 323584-06 \$ 15.00 / 0$
}

ilar tasks, have raised the possibility that the MTL is sometimes needed for working memory (for review, see Ranganath and Blumenfeld, 2005; Graham et al., 2010; but see also Jeneson and Squire, 2012).

In one study (Olson et al., 2006), patients with MTL lesions and controls saw an array of three colored squares and then decided whether or not a designated square in a second array (presented after 4 or $8 \mathrm{~s}$ ) had the same color as the corresponding square in the first array. The poor patient performance in this task was interpreted as a visual working memory deficit, because the material presented was thought to be within visual working memory capacity [i.e., "most people can accurately remember . . . four colors (Luck and Vogel, 1997)," Olson et al., 2006, p. 1093].

Yet, while it is true that young adults typically are able to maintain 3-4 items (Cowan, 2001; Wheeler and Treisman, 2002; Vogel and Machizawa, 2004; Rouder et al., 2008; Fukuda et al., 2010; Jost et al., 2011; Sander et al., 2011), estimates from older adults (as in the Olson et al., 2006 study) are even lower (2-2.5 items; Jost et al., 2011; Sander et al., 2011). In addition, all of these capacity estimates were obtained when memory was assessed after delays of $\sim 1 \mathrm{~s}$, not after delays of $4 \mathrm{~s}$ or longer as in the study by Olson et al. (2006). Accordingly, the requirement in the earlier study to maintain three items for 4 or $8 \mathrm{~s}$ may have exceeded visual working memory capacity. Indeed, based on data presented by Olson et al. (2006) in their Figure 5, we estimated that the controls in that study maintained $\sim 2.3$ colors across the $4 \mathrm{~s}$ delay.

These considerations prompted two key questions. First, do MTL patients and age-matched controls have the same visual working memory capacity (i.e., is their performance the same 
Table 1. Characteristics of memory-impaired patients

\begin{tabular}{|c|c|c|c|c|c|c|c|c|}
\hline \multirow{2}{*}{$\begin{array}{l}\text { Patient } \\
\text { (gender) }\end{array}$} & \multirow{2}{*}{$\begin{array}{l}\text { Age } \\
\text { (years) }\end{array}$} & \multirow{2}{*}{$\begin{array}{l}\text { Education } \\
\text { (years) }\end{array}$} & \multirow[b]{2}{*}{ WAIS-III IQ } & \multicolumn{5}{|l|}{ WMS-R } \\
\hline & & & & Attention & Verbal & Visual & General & Delay \\
\hline GP (M) & 61 & 16 & 98 & 102 & 79 & 62 & 66 & 50 \\
\hline KE (M) & 67 & 13.5 & 108 & 114 & 64 & 84 & 72 & 55 \\
\hline $\mathrm{U}(\mathrm{F})$ & 71 & 12 & 101 & 105 & 83 & 60 & 69 & $<50$ \\
\hline GW (M) & 49 & 12 & 108 & 105 & 67 & 86 & 70 & $<50$ \\
\hline JRW (M) & 45 & 12 & 90 & 87 & 65 & 95 & 70 & $<50$ \\
\hline $\mathrm{RS}(\mathrm{M})$ & 52 & 12 & 99 & 99 & 85 & 81 & 82 & $<50$ \\
\hline
\end{tabular}

WAIS-III is the Wechsler Adult Intelligence Scale-III and WMS-R is the Wechsler Memory Scale-Revised. The WMS-R does not provide numerical scores for individuals who score $<50$. IQ scores for JRW and RS are from the WAIS-R.

after the $1 \mathrm{~s}$ delay typically used to assess capacity)? Second, how well are patients and controls able to retain array sizes within (as well as above) their capacity limit across longer delays? To address these questions, we used a standard change-detection procedure but broadened the parametric space in which visual memory was probed. Specifically, we assessed the ability of patients with MTL damage to maintain 1, 2, 3, 4, or 6 colored squares across delays of $1,3,4$, or $8 \mathrm{~s}$.

\section{Materials and Methods}

Participants. Six memory-impaired patients participated (Table 1). Of these, five have damage thought to be limited to the hippocampus (CA fields, dentate gyrus, and subicular complex). GW and RS became amnesic after drug overdoses and associated respiratory failure. JRW became amnesic after cardiac arrest. KE became amnesic after an episode of ischemia associated with kidney failure and toxic shock syndrome. LJ (the only female) became amnesic during a 6-month period in 1988 with no known precipitating event. Her memory impairment has been stable since that time. Estimates of medial temporal lobe damage were based on quantitative analysis of MR images compared with data from 19 controls (11 for LJ) (Bayley et al., 2005b; Gold and Squire, 2005). GW, RS, JRW, $\mathrm{KE}$, and LJ have an average bilateral reduction in hippocampal volume of $48,33,44,49$, and $46 \%$, respectively (all values $>3$ SDs from the control mean). The volume of the parahippocampal gyrus (temporopolar, perirhinal, entorhinal, and parahippocampal cortices) is reduced by 12 , $1,6,17$, and $-8 \%$, respectively (all values within 2 SDs of the control mean).

One patient (GP) has severe memory impairment resulting from viral encephalitis. GP has demonstrated virtually no new learning since the onset of his amnesia, and during repeated testing over many weeks he does not recognize that he has been tested before (Bayley et al., 2005a). Estimates of medial temporal lobe damage were based on quantitative analysis of MR images and data from four controls. GP has average bilateral reductions in hippocampal volume of $96 \%$. The volume of the parahippocampal gyrus is reduced by $93 \%$. Nine coronal MR images from each of the six patients are available as supplemental material in Shrager et al. (2008).

Twelve healthy controls ( 8 male) served as controls for the memoryimpaired patients. Controls averaged $64.3 \pm 3.0$ years of age and had 14.6 years of education.

Materials and procedure. The materials and procedure were modeled after the change-detection task used by Luck and Vogel (1997) and Olson et al. (2006). Each trial began with a "Get Ready" cue (500 ms) followed by a central fixation cross (300 ms). Next, an array of 1, 2, 3, 4, or 6 colored squares was presented $(200 \mathrm{~ms})$, followed by a second array of squares $(2 \mathrm{~s})$ identical to the first or differing in the color of one square (Fig. 1). The second array was presented after a blank retention interval of $1,3,4$, or 8 s. In the second array, a red box surrounding one of the squares indicated which square might have changed color. The task was to decide by key press whether the cued square had the same color as the corresponding square in the first array or whether it had a different color. In cases where the second array differed from the first (half of the trials), the cued square had a new color that was not present in the first array (i.e., the task was to decide whether a new color had been introduced, not whether a color that was present in the first array was now presented in a new location). Participants could make their response while the array was on the screen $(2 \mathrm{~s})$ or for $2 \mathrm{~s}$ after it had disappeared from the screen. Feedback was provided after each response (the word "correct" in green for correct and the word "incorrect" in red for incorrect).

Seven colors were defined using the RGB color map matrix in Matlab. Color intensity (for red, green, and blue) in the color map ranges from 0 to 255 . The colors were specified as follows: black $[1,1,1]$, white $[254$, $254,254]$, red $[255,0,0]$, green $[0,255,0]$, blue $[0,0,255]$, violet $[200$, $100,255]$, and yellow [254, 254, 115]. The colored squares were presented on a gray background, and each square subtended $1.1^{\circ}$ at a viewing distance of $50 \mathrm{~cm}$. Stimuli were presented in a pseudorandom location in an invisible rectangle that subtended $17.6^{\circ} \times 13.2^{\circ}$ of visual angle in the center of the screen. The minimum distance between squares was $3^{\circ}$ of visual angle.

Participants completed two test sessions. For all but two participants, the two test sessions were separated by at least 1 week (mean $=10.2 \pm$ $1.5 \mathrm{~d}$ ). For patients RS and JRW, the two test sessions were separated by $1-2 \mathrm{~h}$. Each test session consisted of 4 trial blocks of 60 trials each. Half of the trial blocks were given in a verbal load condition and half in a no load condition (in alternating order and counterbalanced across sessions and across participants). In the verbal load condition participants were presented with two digits, presented visually on the computer screen, before the first array. They were asked to hold these digits in memory throughout the trial and then repeat them aloud at the end of the trial. In the no load condition there was no concurrent verbal load. For each condition (verbal load and no load), participants completed a total of 240 trials (4 blocks of 60 trials). Within each block of 60 trials, each unique set size/ delay combination ( 5 set sizes $\times 4$ delays $=20$ unique trial types) was presented in groups of three trials. Thus, each unique trial type was presented three times within 60 trials and 12 times within 240 trials. Each of the 20 trial types was presented in pseudorandom order, with the constraint that the same set size or the same delay was presented for a maximum of 6 trials.

Data analysis. Performance was measured as proportion correct. To further assess capacity, we also converted the hit rates and correct rejection rates to capacity estimates using Cowan's K (Cowan, 2001). Cowan's $K$ is a modification of an earlier method (Pashler, 1988) and estimates the number of items that are successfully held in mind $(K)$ for each of $N$ items in the array that are to be remembered $(K=$ hit rate + correct rejection rate -1$) \times N$, where hit rate is the probability of correctly identifying a change and correct rejection rate is the probability of correctly identifying a no-change. To obtain an estimate of $K$ across array sizes, we adopted the procedure of Alvarez and Cavanagh (2004). For each participant this procedure involves (1) averaging the $K$ estimate across all array sizes, then (2) eliminating the array sizes smaller than this first average or greater than twice this first average, then (3) averaging the $K$ estimates from the remaining array sizes, and (4) iterating this process until the capacity estimate no longer changes. In $19.4 \%$ of cases the estimate did not converge on a single value but oscillated between two or three estimates. In these cases we took the average of these estimates. This procedure limits the array sizes included in the capacity estimate to array sizes near each participant's capacity.

Invalid trials, in which participants did not respond within the response period, were rare (controls: $0.5 \%$ of trials; patients: $1.2 \%$ of trials) and were not included in the analysis. Performance measures in the verbal load condition were based on all trials, regardless of performance on the concurrent digit task.

For repeated-measures ANOVA, Greenhouse-Geisser adjusted $p$-values were reported when sphericity was violated (Greenhouse and Geisser, 1959), along with the adjusted degrees of freedom.

\section{Results}

We first examined the effect of condition (no verbal load vs verbal load). As can be appreciated in Figure 2, the requirement in the verbal load condition to hold two digits in mind across each trial compromised both patient and control performance for the larger, but not the smaller, array sizes. Thus, an overall ANOVA [condition $\times$ array size $\times$ delay $\times$ group (controls vs hippocam- 
pal patients)] revealed an effect of group $\left(F_{(1,15)}=6.6 ; p<0.05\right)$, condition $\left(F_{(1,15)}=\right.$ 25.2; $p<0.001)$, and array size $\left(F_{(4,60)}=\right.$ 95.7; $p<0.001)$. There was also a condition $\times$ array size interaction $\left(F_{(2.5,37.3)}=\right.$ 3.6; $p<0.05)$ and a group $\times$ array size interaction $\left(F_{(4,60)}=2.7 ; p<0.05\right)$. In addition, the effect of condition was significant for both groups (controls: $F_{(1,11)}=9.6 ; p<$ 0.05 ; hippocampal patients: $F_{(1,4)}=14.7$; $p<0.05)$.

Given these effects of verbal load, the main analyses assessed performance separately for the no load and the verbal load conditions. Scores for the three different delays $(3,4$, and $8 \mathrm{~s})$ were averaged to assess performance at the longer delays because, for both conditions, a delay (3, 4, or $8 \mathrm{~s}) \times$ array size $\times$ group ANOVA revealed no effect of delay and no delay $X$ group interaction. For completeness, Cowan's $K$ estimates for each of the four delays are provided in Table 2.

For each condition, we asked whether patients and controls performed similarly at the $1 \mathrm{~s}$ delay typically used to assess capacity and whether their capacity estimates were the same. We also asked how well patients and controls were able to maintain information across delays longer than the $1 \mathrm{~s}$ delay, and how their performance might vary as a function of array size.

At the $1 \mathrm{~s}$ delay typically used to assess capacity, patients and controls performed similarly in both the no load $(p>0.9)$ and the verbal load condition ( $p>0.05)$ (Figs. 3 and 4, respectively, panels to the left).

The corresponding Cowan's $K$ estimates were also similar (Table $2, p$-values $>0.2$ ). At the longer delays, by contrast, patients performed worse than controls in both conditions (no verbal load, Fig. 3, right: $\left(F_{(1,15)}=4.9 ; p<0.05\right)$; verbal load, Fig. 4, right: $\left.\left(F_{(1,15)}=8.3 ; p<0.05\right)\right)$. The $K$ estimates were also lower for the patients than for the controls at the longer delays (No load: $t_{(15)}=$ 2.9, $p<0.05$; verbal load: $\left.t_{(15)}=2.8, p<0.05\right)$.

The group effect at the longer delays was due to the fact that the controls performed better than the hippocampal patients at the larger array sizes (3, 4, and 6 items; Figs. 3 and 4, panels to the right). With smaller array sizes ( 1 and 2 items), the patients performed like controls even after longer delays. Patient GP with larger MTL lesions was impaired at the longer delays like the hippocampal patients. At the short delay, his performance was good in the no load condition (except for array size 4; Table 2; Fig. 3) and lower than controls in the verbal load condition $(p<0.01$; Table 2; Fig. 4$)$.

Interestingly, in the no load condition, with larger array sizes, the difference between patients and controls at long delays occurred because controls (but not patients) performed better after long delays than after the short delay (Table 2; Fig. 3). This observation was documented by a delay (short versus long) $X$ array size interaction for controls $\left[F_{(2.0,22.4)}=5.6 ; p<0.05\right]$ but not for the hippocampal patients (Fig. 3). Furthermore, the $K$ estimates for controls in the no load condition were significantly higher at
Table 2. Cowan's K estimates of visual working memory capacity

\begin{tabular}{|c|c|c|c|c|c|}
\hline & $\begin{array}{l}\text { Short delay } \\
\text { (1 s) }\end{array}$ & $3 \mathrm{~s}$ & $4 s$ & $8 \mathrm{~s}$ & $\begin{array}{l}\text { Longer delays } \\
(3,4 \text {, and } 85)\end{array}$ \\
\hline \multicolumn{6}{|l|}{ No verbal load } \\
\hline Controls $(n=12)$ & $2.62(0.2)$ & $3.66(0.4)$ & $3.05(0.2)$ & $3.28(0.4)$ & $3.33(0.3)$ \\
\hline $\mathrm{H}(n=5)$ & $2.46(0.2)$ & $1.47(0.5)^{*}$ & $2.30(0.3)$ & $1.87(0.2)^{*}$ & $1.88(0.3)^{*}$ \\
\hline $\operatorname{MTL}(n=1)$ & 2.11 & 1.77 & 1.75 & 2.54 & 2.02 \\
\hline \multicolumn{6}{|l|}{ Verbal load } \\
\hline Controls $(n=12)$ & $2.32(0.2)$ & $2.60(0.2)$ & $2.48(0.1)$ & $2.43(0.2)$ & $2.50(0.1)$ \\
\hline $\mathrm{H}(n=5)$ & $1.93(0.2)$ & $1.83(0.4)^{*}$ & $1.73(0.3)^{*}$ & $1.36(0.4)^{*}$ & $1.64(0.4)^{*}$ \\
\hline $\operatorname{MTL}(n=1)$ & 1.50 & 1.50 & 1.75 & 1.83 & 1.69 \\
\hline
\end{tabular}

The mean (and SEM) overall capacity estimates for each of the four delays and the three longer delays combined are shown. The capacity estimates for the short (1s) delay and the longer delays correspond to the data presented in the left and the right panels of Figure 3 (no verbal load) and Figure 4 (verbal load), respectively. Asterisks indicate significant difference between patients and controls $(p<0.05)$.

the longer delays than at the $1 \mathrm{~s}$ delay (Table $2 ; t_{(11)}=2.6, p<$ $0.05)$. By contrast, the performance of the hippocampal patients at the $1 \mathrm{~s}$ delay was no different from their performance at the longer delays (Fig. 3; all $p$-values for array sizes 3, 4, and 6>0.15), and their $K$ estimate for the longer delays was marginally smaller than their $K$ estimate for the short delay (Table $2 ; t_{(4)}=2.3, p=$ 0.08 ). Although these effects for controls (better performance at the longer delays than at the short delay) did not obtain in the verbal load condition, there was a small numerical trend even in that condition for higher capacity estimates after the long delays 


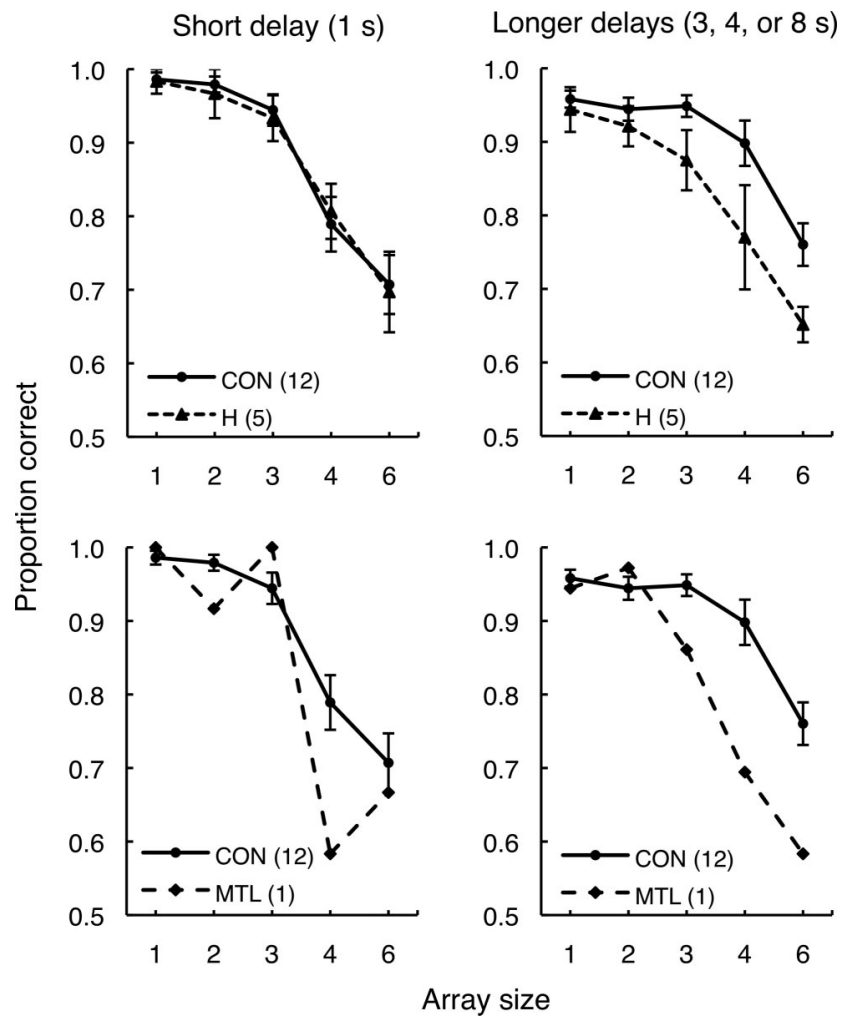

Figure 3. Performance as a function of array size (no verbal load). The data show performance at the $1 \mathrm{~s}$ delay (left) and at the three longer delays (right). Controls (CON) are compared with hippocampal patients (H; top) and to patient GP with large MTL lesions (MTL; bottom). Error bars indicate SEM.

than after the short delay (Table 2). As in the no load condition, the performance of the patients at the $1 \mathrm{~s}$ delay was similar to their performance at the longer delays (Fig. 4 ; all $p$-values for array sizes 3,4 , and $6>0.35$ ), and their $K$ estimates were also similar at the short and at the longer delays (Table 2; $p=0.3$ ).

Although our main analyses focused on performance on the visual change-detection task, we also examined performance on the digit task in the verbal load condition. Overall, the hippocampal patients made more digit errors than controls (errors on $14.6 \pm 6.1 \%$ of trials compared with $3.5 \pm 1.6 \%$ of trials, respectively). The MTL patient performed as well as controls (errors on $1.7 \%$ of trials). Interestingly, both patients and controls exhibited an increase in the number of digit errors with increasing array size. The hippocampal patients made errors on 10.0, 15.4, 15.8, 13.8 , and $17.9 \%$ of trials for array sizes $1,2,3,4$, and 6 , respectively (collapsed across the four delays). The corresponding scores for controls were 1.9, 3.3, 3.6, 3.5, and 5.2\% (see also Morey and Cowan, 2004, 2005, for similar effects of total memory load on amodal interference). The greater vulnerability to interference from concurrent verbal load in patients compared with controls likely accounts for some of the difference in patient and control performance observed in the verbal load condition (see Discussion).

\section{Discussion}

We used a standard change-detection task (Luck and Vogel, 1997) to assess the role of the hippocampus and related MTL structures in retention of simple visual information. Patients with MTL damage (five with lesions restricted to the hippocampus; one with larger MTL lesions) and age-matched controls at-

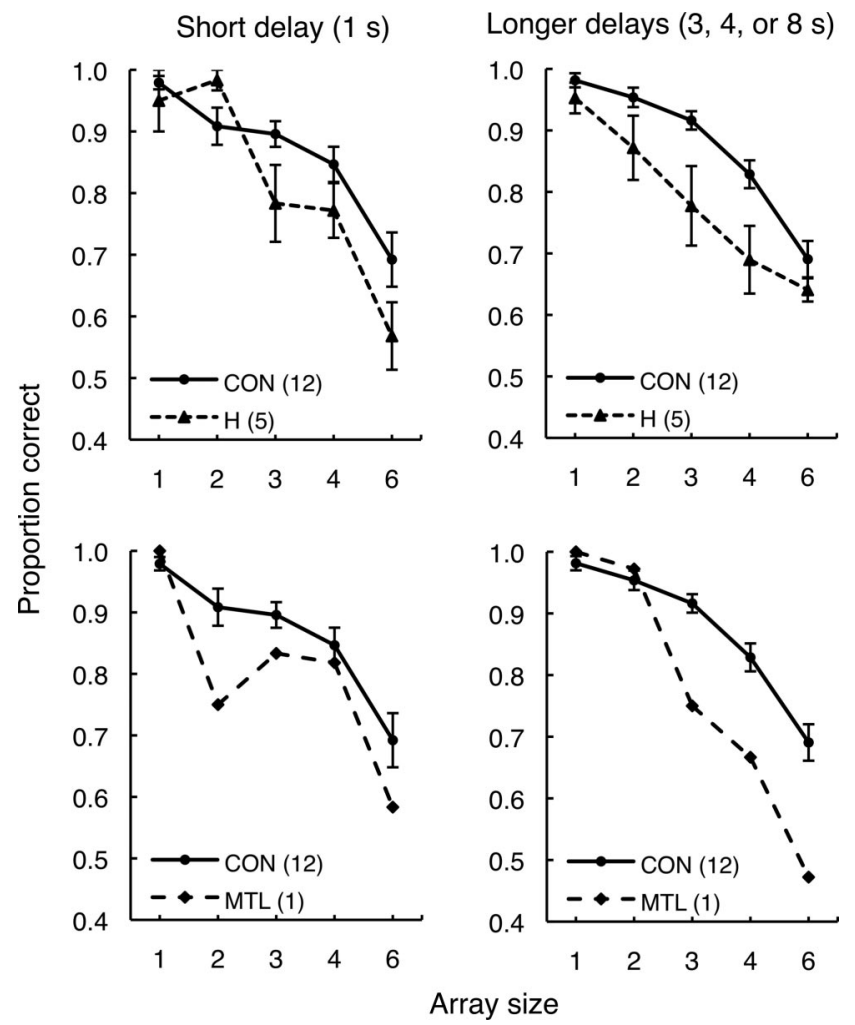

Figure 4. Performance as a function of array size (with a verbal load). The data show performance at the $1 \mathrm{~s}$ delay (left) and at the three longer delays (right). Controls (CON) are compared with hippocampal patients ( $\mathrm{H}$; top) and to patient GP with large medial temporal lobe lesions (MTL; bottom). Error bars indicate SEM.

tempted to remember 1, 2, 3, 4, or 6 colored squares across delays of $1,3,4$, or $8 \mathrm{~s}$. At the $1 \mathrm{~s}$ delay (in the no load condition) typically used to assess visual working memory capacity (Luck and Vogel, 1997; Todd and Marois, 2004; Vogel and Machizawa, 2004; Jost et al., 2011; Sander et al., 2011), patients performed as well as controls at all array sizes, even when performance was not at ceiling (Fig. 3). The corresponding capacity estimates for patients and controls at the $1 \mathrm{~s}$ delay were also approximately the same (2-2.5 items with no verbal load; Table 2) and similar to those obtained from older adults in two recent studies (Jost et al., 2011; Sander et al., 2011). (Note that in these two studies visual working memory capacity was assessed without a concurrent verbal load.) At the longer delays, patients performed as well as controls when the number of items to be maintained was within this capacity limit ( 1 and 2 items), and they performed worse than controls when the number of items to be maintained exceeded this limit (3, 4, or 6 items).

Our findings replicated the previous finding (Olson et al., 2006) that patients with MTL damage are impaired at remembering 3 colored squares after $4 \mathrm{~s}$. In the earlier study, as well as in a number of other studies assessing short-term retention of visual information in patients with MTL damage, impaired performance has been interpreted to mean that the MTL is critical for at least some kinds of working memory (for reviews representing this view, see Ranganath and Blumenfeld, 2005; Graham et al., 2010). Yet, our findings at the $1 \mathrm{~s}$ delay, across all array sizes (and without verbal load), demonstrate intact visual working memory capacity after MTL damage.

In the verbal load condition, both controls and patients performed worse than in the no load condition (Fig. 2). There was a tendency for the patients to be affected by the verbal load 
more than the controls, though the interaction of group $\times$ load condition did not reach significance $(p=0.11)$. Interestingly, this tendency was also evident for patient GP (Table 2; Fig. 2). GP made only $1.7 \%$ errors in the verbal digit task (controls, 3.5\%; hippocampal patients, $14.6 \%$ ). His low scores in the verbal load condition may therefore reflect the particularly strong attention he directed to the digit task, thereby impairing his performance on the change-detection task itself.

Working memory involves the process of active maintenance of a limited amount of information and cannot be operationally defined in terms of any particular retention interval. If the amount of information to be maintained exceeds visual working memory capacity, or if attention is diverted, then performance depends in part on long-term memory, even at short retention intervals (Drachman and Arbit, 1966; Jeneson and Squire, 2012; see also Brady et al., 2011). In studies where the contribution of working memory and long-term memory to task performance have been formally assessed, patients with MTL damage exhibited intact performance so long as working memory was sufficient to support performance, and they were impaired only when long-term memory was also needed to support performance (Shrager et al., 2008; Jeneson et al., 2010; for a review of both patient data and neuroimaging data related to this issue, see Jeneson and Squire, 2012).

For example, in Jeneson et al. (2010), the task required participants to maintain up to seven object-location associations across a $1 \mathrm{~s}$ delay. MTL patients performed similarly to controls when only a small number of object-location associations needed to be remembered, but they exhibited an abrupt decline in performance when as many as 3-4 object locations needed to be remembered. The marked discontinuity in patient performance as they moved from smaller to larger set sizes occurred at approximately the same set size that first produced errors in controls. Presumably, controls began making errors at this point because the material now exceeded their working memory capacity. These findings suggested that active maintenance is intact in patients with MTL damage. The patients were impaired only when the task exceeded working memory capacity such that long-term memory now benefited performance. The findings in the current study are consistent with the findings of Jeneson et al. (2010). We propose that patients were impaired only when the amount of information to be remembered exceeded visual working memory capacity (i.e., at longer delays and at larger array sizes).

What might account for the finding that, in the no load condition, controls performed better after the longer delays than after the short delay? It seems unlikely that, at the short delay but not at the longer delays, the presentation of the second array disrupted transfer of the sample array into visual working memory. It has been shown that transfer into visual working memory, measured by varying the time between presentation of the sample and an interrupting mask, does not continue beyond $200-300 \mathrm{~ms}$ (or $50 \mathrm{~ms}$ per item) after the onset of the sample array (Vogel et al., 2006). One intriguing possibility is that controls benefited from longer delays because these conditions enabled them to recode, elaborate, and stabilize the visual information using longterm memory (e.g., by verbally rehearsing the colors during the delay). It has been noted that longer delays ( $>1 s$ ) "not only underestimate VSTM [visual working memory] capacity because of memory degradation (Phillips, 1974), but also favor the recruitment of rehearsal mechanisms and verbal/abstract recoding of the visual material (Coltheart, 1972)" (Todd and Marois, 2004, p. 751). Thus, at the longer delays, as participants realize that they will need to retain information for longer than $\sim 1 \mathrm{~s}$, they might engage in effortful rehearsing to retain as much information as possible. As a result, estimates of capacity obtained by controls at these longer delays may reflect not only the limits of working memory but also the contribution of long-term memory. Consistent with this idea, it is interesting that in the verbal load condition, which could be expected to minimize verbal rehearsal and thereby reduce the contribution of long-term memory, controls exhibited little or no improvement in performance at the longer delays compared with the $1 \mathrm{~s}$ delay (Table 2; Fig. 4).

In studies of the neural correlates of visual working memory and visual working memory capacity, the key areas that have been implicated are, not MTL structures, but intraparietal sulcus, visual cortex, and the prefrontal cortex. For example, fMRI activity in the intraparietal sulcus and areas of occipital cortex has been found to increase with increasing array size, reaching asymptote at the point where capacity is exceeded (Todd and Marois, 2004, 2005; Xu and Chun, 2006). In addition, numerous findings suggest that maintenance of information in working memory is supported by sustained activity in the various brain areas that process or encode the to-be-remembered information (Jonides et al., 2005; Pasternak and Greenlee, 2005; Postle, 2006). For example, maintenance of motion direction was associated with sustained activity in area MT (Bisley and Pasternak, 2000; Bisley et al., 2004), and working memory for faces was associated with sustained activity in posterior fusiform gyrus (Ranganath et al., 2004). Using a multivoxel pattern analysis, Serences et al. (2009) observed stimulus-specific delay activity in primary visual cortex (V1) during maintenance of the orientation or the color of a multifeature object. This activity was qualitatively similar to the activity observed in $\mathrm{V} 1$ during processing of the relevant sensory information (i.e., orientation or color). Other work has also identified the importance of prefrontal cortex (Goldman-Rakic, 1995; Fuster, 2008). Cells in prefrontal cortex are maximally active during the delay portion of the delayed-response task (Fuster and Alexander, 1971). In addition, patients with unilateral prefrontal cortex lesions were impaired on a lateralized change-detection task when arrays of colored squares were presented to the visual field contralateral to the lesion (i.e., when the information was presented to the damaged hemisphere) (Voytek and Knight, 2010). One possibility is that maintenance of visual information in working memory is supported by sustained activity in the same sensory areas that are involved in the encoding of the memoranda, and that the prefrontal cortex supports working memory by directing attention to task-relevant sensory signals (Postle, 2006).

In summary, we demonstrated that visual working memory capacity is intact after MTL damage (without a concurrent verbal load) and that MTL damage impairs performance after delays of 3,4 , and 8 s only when the information to be held in mind exceeds the capacity limit of visual working memory $(2-2.5$ items in older adults). At these longer delays, patients performed as well as controls at array sizes below the capacity limit ( 1 or 2 items), and they performed worse than controls only at array sizes that exceeded the capacity limit $(3,4$, or 6 items). Together with other findings (see Jeneson and Squire, 2012), these findings suggest that MTL damage impairs performance only under conditions where working memory is insufficient to support good performance. In these cases, controls gain an advantage over patients because performance can be supported by long-term memory in addition to working memory. 


\section{References}

Alvarez GA, Cavanagh P (2004) The capacity of visual short-term memory is set both by visual information load and by number of objects. Psychol Sci 15:106-111.

Baddeley AD, Hitch GJ (1974) Working memory. In: The psychology of learning and motivation: advances in research and theory (Bower GH, ed), pp 47-89. Academic: New York.

Baddeley AD, Warrington EK (1970) Amnesia and the distinction between long- and short-term memory. J Verb Learn Verb Behav 9:176-189.

Bayley PJ, Frascino JC, Squire LR (2005a) Robust habit learning in the absence of awareness and independent of the medial temporal lobe. Nature 436:550-553.

Bayley PJ, Gold JJ, Hopkins RO, Squire LR (2005b) The neuroanatomy of remote memory. Neuron 46:799-810.

Bisley JW, Pasternak T (2000) The multiple roles of visual cortical areas MT/ MST in remembering the direction of visual motion. Cereb Cortex 10:1053-1065.

Bisley JW, Zaksas D, Droll JA, Pasternak T (2004) Activity of neurons in cortical area MT during a memory for motion task. J Neurophysiol 91:286-300.

Brady TF, Konkle T, Alvarez G (2011) A review of visual memory capacity: beyond individual items and toward structured representations. J Vis 11:1-34.

Coltheart M (1972) Visual information-processing. In: New horizons in psychology (Dodwell PC, ed), pp 62-85. Harmondsworth: Penguin.

Cowan N (2001) The magical number 4 in short-term memory: a reconsideration of mental storage capacity. Behav Brain Sci 24:87-185.

Drachman DA, Arbit J (1966) Memory and the hippocampal complex. II. Is memory a multiple process? Arch Neurol 15:52-61.

Fukuda K, Awh E, Vogel EK (2010) Discrete capacity limits in visual working memory. Curr Opin Neurobiol 20:177-182.

Fuster JM (2008) The prefrontal cortex, Ed 4. London: Academic.

Fuster JM, Alexander GE (1971) Neuron activity related to short-term memory. Science 173:652-654.

Gold JJ, Squire LR (2005) Quantifying medial temporal lobe damage in memory-impaired patients. Hippocampus 15:79-85.

Goldman-Rakic PS (1995) Architecture of the prefrontal cortex and the central executive. Ann N Y Acad Sci 769:71-83.

Graham KS, Barense MD, Lee ACH (2010) Going beyond LTM in the MTL: a synthesis of neuropsychological and neuroimaging findings on the role of the medial temporal lobe in memory and perception. Neuropsychologia 48:831-853.

Greenhouse SW, Geisser S (1959) On methods in the analysis of profile data. Psychometrika 24:95-112.

Jeneson A, Squire LR (2012) Working memory, long-term memory, and medial temporal lobe function. Learn Mem 19:15-25.

Jeneson A, Mauldin KN, Squire LR (2010) Intact working memory for relational information after medial temporal lobe damage. J Neurosci 30:13624-13629.

Jonides J, Lacey SC, Nee DE (2005) Processes of working memory in mind and brain. Curr Dir Psychol Sci 14:2-5.

Jost K, Bryck RL, Vogel EK, Mayr U (2011) Are old adults just like low working memory young adults? Filtering efficiency and age differences in visual working memory. Cereb Cortex 21:1147-1154.

Luck SJ, Vogel EK (1997) The capacity of visual working memory for features and conjunctions. Nature 390:279-281.

Milner B (1972) Disorders of learning and memory after temporal lobe lesions in man. Clin Neurosurg 19:421-446.

Morey CC, Cowan N (2004) When visual and verbal memories compete: evidence of cross-domain limits in working memory. Psychon Bull Rev 11:296-301.

Morey CC, Cowan N (2005) When do visual and verbal memories conflict? The importance of working-memory load and retrieval. J Exp Psychol Learn 31:703-713.

Olson IR, Moore KS, Stark M, Chatterjee A (2006) Visual working memory is impaired when the medial temporal lobe is damaged. J Cogn Neurosci 18:1087-1097.

Pashler H (1988) Familiarity and the detection of change in visual displays. Percept Psychophys 44:369-378.

Pasternak T, Greenlee MW (2005) Working memory in primate sensory systems. Nat Rev Neurosci 6:97-107.

Phillips WA (1974) On the distinction between sensory storage and shortterm visual memory. Percept Psychophys 16:283-290.

Postle BR (2006) Working memory as an emergent property of the mind and brain. Neuroscience 139:23-38.

Ranganath C, Blumenfeld RS (2005) Doubts about double dissociations between short- and long-term memory. Trends Cogn Sci 9:374-380.

Ranganath C, DeGutis J, D’Esposito M (2004) Category-specific modulation of inferior temporal activity during working memory encoding and maintenance. Cogn Brain Res 20:37-45.

Rouder JN, Morey RD, Cowan N, Zwilling CE, Morey CC, Pratte MS (2008) An assessment of fixed-capacity models of visual working memory capacity. Proc Natl Acad Sci U S A 105:5975-5979.

Sander MC, Werkle-Bergner M, Lindenberger U (2011) Contralateral delay activity reveals life-span age differences in top-down modulation of working memory contents. Cereb Cortex 21:2809-2819.

Serences JT, Ester EF, Vogel EK, Awh E (2009) Stimulus-specific delay activity in human primary visual cortex. Psychol Sci 20:207-214.

Shrager Y, Levy DA, Hopkins RO, Squire LR (2008) Working memory and the organization of brain systems. J Neurosci 28:4818-4822.

Todd JJ, Marois R (2004) Capacity limit of visual short-term memory in human posterior parietal cortex. Nature 428:751-754.

Todd JJ, Marois R (2005) Posterior parietal cortex activity predicts individual differences in visual short-term memory capacity. Cogn Affective Behav Neurosci 5:144-155.

Vogel EK, Machizawa MG (2004) Neural activity predicts individual differences in visual working memory capacity. Nature 428:748-751.

Vogel EK, Woodman GF, Luck SJ (2006) The time course of consolidation in visual working memory. J Exp Psychol Hum 32:1436-1451.

Voytek B, Knight RT (2010) Prefrontal cortex and basal ganglia contributions to visual working memory. Proc Natl Acad Sci USA 107:18167-18172.

Wheeler ME, Treisman AM (2002) Binding in short-term visual memory. J Exp Psychol Gen 131:48-64.

Xu Y, Chun MM (2006) Dissociable neural mechanisms supporting visual short-term memory for objects. Nature 440:91-95. 